\title{
LA FORMACIÓN EN ORIENTACIÓN PROFESIONAL DE LOS JÓVENES EUROPEOS
}

\author{
THE TRAINING ON VOCATIONAL GUIDANCE OF \\ EUROPEAN YOUNG PEOPLE
}

\author{
Luis Sobrado* \\ Universidad de Santiago de Compostela
}

\section{RESUMEN}

En este artículo se plantean las finalidades y el rol de la Orientación Profesional así como sus posibilidades formativas en relación con la juventud europea.

Se exponen además las funciones de los Servicios de Orientación Vocacional y las acciones formativas en el marco orientador, referidas a la integración en el currículo escolar, la elección vocacional y las actividades de transición académica y de ésta a la vida laboral del sujeto, con referencia especial a su inserción profesional.

Este enfoque de la formación en Orientación Profesional se realiza desde la necesidad de articular estrechamente el mundo de la Educación y el del Trabajo, todavía bastante descoordinados en la realidad actual.

Palabras clave: Empleo, joven, formación, servicios de Orientación, transición, inserción, profesión, orientación vocacional, Unión Europea, mercado laboral, colocación, integración.

\footnotetext{
ABSTRACT

This paper defines the aims and the role of Vocational Guidance as well as the possibilities open for the training of European young people.

We also explain how Vocational Guidance works as well as the training methods used in the guidance framework, with reference to the school curriculum, vocational choice and to the activities of academic transition and from here to the individual's working life with special reference to professional placement.

This Vocational Guidance training focus comes from the need to join the worlds of Education and work which, in today's world, are basically rather uncoordinated.

* Catedrático de Universidad de Diagnóstico y Orientación, Departamento de Métodos y Técnicas de Investigación en Ciencias del Comportamiento y de la Educación de la Universidad de Santiago de Compostela. Líneas de investigación: Diagnóstico de necesidades y competencias orientadoras; Desarrollo profesional de Orientadores Escolares y Laborales y Evaluación de Servicios y Programas de Orientación.
} 
Key words: Employment, young person,training, guidance services, placement profession,vocational guidance, European Union, work market, employment integration.

\section{Introducción}

El desarrollo creciente del desempleo en los distintos países de la Unión Europea en los últimos 25 años donde se pasó de un 3,7\% en 1975 a un 12\% aproximadamente en 1999, coloca en un primer plano una serie de acciones a realizar para ayudar a las personas a atravesar esta etapa de crisis.

Las causas del desempleo son sin duda estructurales y macroeconómicas pero también existen carencias individuales en el ámbito de la formación, cualificaciones y competencias profesionales, etc., que inciden en este problema social.

No obstante no todos los grupos de sujetos son iguales delante del fenómeno del desempleo, de las dificultades de inserción profesional, etc. La juventud es uno de los sectores más afectados y, por eso, debe ser objeto especial de atención en el momento de intervenir en el mercado de trabajo. Representa una pieza importante en un país pues ella constituye la futura población activa.

Además de la educación inicial, la formación complementaria y los primeros años de vida laboral activa condicionan mucho las posibilidades de inserción profesional estable y de éxito en la carrera profesional.

El riesgo de la futura exclusión social, cultural o profesional de los jóvenes está muy determinada por los itinerarios de formación y orientadores seguidos por ellos.

La Unión Europea con vistas a la conservación de la cohesión social, la lucha contra el desempleo, especialmente el de larga duración y para prevenir los riesgos de la exclusión social, económica, cultural y profesional demostró un gran interés proyectado en los siguientes documentos:

- Libro Blanco de la Comisión Europea de 1993 sobre Crecimiento, Competitividad y Empleo.

- Documento sobre la Enseñanza y Aprendizaje: hacia la sociedad cognitiva en el año 1995.

- El capítulo social del tratado de Maastricht.

Más recientemente en 1997 las directrices del Consejo para el Empleo de Luxemburgo, resaltan una serie de acciones a emprender en los Estados miembros de la Unión Europea, y una de ellas enfatiza la necesidad de mejora de la capacidad de inserción profesional de los adultos y los jóvenes.

En la cumbre de Luxemburgo se recoge explícitamente la necesidad de atención especial a los jóvenes con más de seis meses de desempleo, a través de actividades de formación, reconversión y experiencia profesional, de empleo o de cualquiera otra medida para posibilitar la inserción laboral, entre ellas la orientación y consejo profesional.

Asimismo se trata de ofrecer a los trabajadores adultos con más de un año de paro una serie de acciones de formación y también de acompañamiento individual de orientación profesional. 
La inserción profesional de la juventud está vinculada a la transición escuela-empleo y ello ocupa el centro de interés de los programas europeos Leonardo da Vinci y Sócrates.

En el año 2000 en el Consejo Europeo de Lisboa se consideró que la cohesión social debe ser parte integrante de una economía basada en el saber.

Otras instituciones internacionales preocupadas por este ámbito es la Fundación Europea de la Vida y el Trabajo que en 1991 creó el programa de investigación-acción Euroconsel; igualmente se destacan los informes de la OCDE, la OIT, del CEDEFOP, etc. En todos estos organismos se subraya una actitud de refuerzo del rol que deben asumir los servicios de orientación y de consejo profesional para el empleo y de los itinerarios formativos y profesionales y proyectos de vida especialmente de los jóvenes.

La Unión Europea posee un potencial humano bien cualificado globalmente, y sistemas de protección social esenciales para la transición hacia una sociedad basada en el crecimiento.

\section{Posibilidades de la Orientación Vocacional}

Diversas transformaciones esenciales se generan en el presente en diferentes países de Europa y afectan a varios ámbitos sociales y económicos como son las repercusiones de las nuevas tecnologías que cambian el trabajo y la necesidad de cualificaciones muy superiores que precisan los trabajadores respecto a las que se exigían tradicionalmente.

Por otra parte los empresarios se quejan de que los centros educativos no forman a los alumnos con competencias ajustadas a sus demandas.

Esta realidad genera situaciones de desempleo por la carencia de ajuste entre oferta y demanda laboral, de tal modo que los poderes públicos se van concienciando de la necesidad de que todos los ciudadanos aprendan a lo largo de la vida, objetivo aún no conseguido actualmente.

Todo esto representa que las personas deben tomar decisiones para formarse para las distintas modalidades de trabajo, de tal modo que los programas educativos deben ampliarse y diversificarse para adecuarse a unos empleos muy especializados, de corta duración y menos seguros que en épocas pasadas.

Ante la existencia creciente de situaciones complejas en el mercado laboral las decisiones políticas no garantizan que éste resuelva la adecuación entre la oferta y la demanda de trabajo y la necesidad de competencias y cualificaciones profesionales idóneas, ni que las elecciones ocupacionales de carácter personal no se hagan al azar en muchas situaciones.

Todos los países poseen la necesidad de que sus ciudadanos hagan el mejor uso posible de sus capacidades y aptitudes, y aseguren la relevancia de las conexiones entre formación, orientación y empleo.

Sin embargo estas relaciones pueden ser difíciles de fijar si los sistemas de enseñanza, asesoramiento y colocación evolucionan por separado como así fue tradicionalmente. Cuando no hay interacción entre estos tres sectores se generan una serie de inadecuaciones y disfunciones en los jóvenes y adultos dirigidos en muchas ocasiones a elecciones desajustadas de carácter laboral que condicionan una realidad profesional. 
Uno de los instrumentos para obviar estas anomalías es la disposición de servicios de orientación para los jóvenes y adultos que se ven en la obligación de elegir su futuro laboral.

Como señala Watts (1996) la orientación de más calidad no resuelve todos los problemas de empleo, pero diversas actividades de orientación escolar y profesional pueden prestar buenos servicios a las políticas activas del mismo y ayudar a sacar la mejor adecuación posible de las aptitudes e intereses profesionales para conseguir una vida más satisfactoria.

Un estudio da OCDE (1998) muestra que la opinión pública cuida como una de las grandes funciones de los centros docentes la orientación escolar y profesional. En otro trabajo también de la OCDE (1999) se constata que una formación completa y costosa puede resultar inútil si no va acompañada de una orientación idónea.

La orientación profesional es todavía un sector descuidado pero puede ser uno de los componentes significativos de las políticas activas de empleo en los próximos años.

Entre las finalidades y metas de la Orientación Vocacional figuran las siguientes:

$1^{\text {a }}$. Analizar los servicios e instituciones de orientación profesional destinados a los jóvenes que deben elegir estudios.

$2^{\mathrm{a}}$. Informar sobre las necesidades laborales de la juventud en un mercado laboral en rápida mutación.

$3^{\mathrm{a}}$. Exponer la problemática de las transiciones en diversas etapas de la vida del joven: tránsito de la escuela obligatoria a la vida profesional, de las diferentes transiciones académicas (formación profesional, enseñanza superior,...) y de la del graduado universitario al ámbito ocupacional.

\section{Posibilidades de la Orientación Profesional}

La Orientación se entiende actualmente como una actividad que se refiere a un conjunto de informaciones, consejos y asesoramientos en el ámbito académico, profesional, etc.

Desde la perspectiva educativa se trata de poner a disposición de los alumnos una serie de experiencias que los ayuden a comprenderse a sí mismos, a interpretar una serie de procesos y procedimientos destinados a realizar ciertas metas personales y escolares, y a comprender además el mundo que los rodea.

Gysbers y Henderson (1998) señalan que un programa de orientación educativa y profesional debe constar de una serie de aspectos como son la planificación de la carrera de carácter personal, unos servicios para dar ayuda especial a los alumnos con dificultades, un sistema de apoyo con un plan de actividades, un programa orientador y unas tareas de asesoramiento garantizadas por personal especializado.

La orientación no se sitúa únicamente en el cuadro de los centros escolares. El término recoge muchas medidas de consejo y de apoyo ofrecidos a los jóvenes y a los adultos para ayudarles al máximo desarrollo de sus posibilidades formativas (a escoger los cursos y los programas que les interesen, a asesorarles de modo eficiente a superar obstáculos y dificultades, a ayudarles a tomar decisiones realistas en el ámbito de la formación profesional, en la elección de la profesión y del empleo y a ponerlas en práctica, etc.). 
Los servicios de orientación profesional abarcan normalmente tres ámbitos, siendo conscientes de que los términos «orientación» o «consejo» tienen significaciones diversas según países, escuelas científicas, etc. Son los siguientes:

a) Educativo, que se refiere al aspecto de formación académica de la persona y también a la ayuda para conseguir habilidades sociales, de trabajo..., escoger materias o itinerarios ajustados a sus necesidades, elegir el tipo de centro donde escolarizarse, intentar superar las dificultades de aprendizaje, etc.

b) Personal y Social, que versa sobre aspectos que pueden ser de carácter afectivo (dificultades familiares, valores, problemas de comportamiento, etc.) o de naturaleza social (asesoramiento en el sector de contratos laborales, ayuda financiera, problemas jurídicos...).

c) Profesional, que trata sobre las expectativas laborales, ayuda para escoger una función ocupacional en la vida activa, la colocación y generalmente a facilitar el pase de la formación a la vida activa.

Un sistema de orientación profesional de calidad posee las siguientes posibilidades para los ámbitos que se expresan:

$1^{\circ}$. Centros formativos, pues sus programas serán más eficaces si el alumnado sigue los cursos que responden a sus necesidades y de esta forma las tasas de abandono pueden reducirse.

$2^{\circ}$. Poderes públicos, ya que una buena orientación facilita utilizar mejor los recursos humanos.

$3^{\circ}$. Los empresarios, pues les ayuda a encontrar a los trabajadores que precisan con el tipo de formación que necesitan, o que les sirve para mejorar sus competencias y motivaciones, etc.

$4^{\circ}$. Las personas en concreto, pues las ayuda a diseñar posibles itinerarios formativos y/o profesionales en contextos de diferentes posibilidades educativas y laborales, y a realizar elecciones realistas y claras.

Todas estas categorías deben posibilitar la igualdad de oportunidades y obviar la existencia de elementos perjudiciales debidos al contexto étnico, sexo, lengua o estatus socioeconómico. Se trata de desenvolver una orientación provista de efectividad que identifique las posibilidades existentes en el sector de la enseñanza, de la formación continua y del empleo y comenzar a construir itinerarios, elaborados por los propios jóvenes.

El proyecto INES de la OCDE (1995) señala que la opinión pública de los países que la conforman reconoce las posibilidades de la información y orientación profesional en la escuela y se declara a favor de ella. Un $80 \%$ de población de 12 países de la OCDE estima que la información y los consejos al alumnado sobre profesiones es uno de los roles esenciales de la escuela. Así en Austria, un 93\% estima que la orientación escolar y profesional es la acción más importante de los centros docentes. Le sigue Francia y Escocia con un $89 \%$ de la población y de las empresas que la consideran muy importante, según datos facilitados por el Scottish Office Education Departament (1995).

Walz (1987) destaca que la Orientación Profesional posee un gran realce por la ayuda a las personas en su proyecto, estilo de vida y por la relación estrecha entre el perfil de las carreras profesionales y la evolución personal. 
La orientación profesional debe tener en cuenta los valores, los objetivos y las creencias personales y la necesidad de una planificación sistemática y una responsabilidad personal en el desarrollo de la carrera académico-profesional.

\section{Destinatarios de la Orientación Vocacional}

En su conjunto la Orientación Educativa es ofertada especialmente a los jóvenes, habitualmente en los Centros docentes, y a veces también por otras instituciones.

La Orientación Profesional es impartida actualmente en diferentes establecimientos educativos (escuelas, universidades, centros formativos) y también por instituciones sociales (agencias de empleo, empresas, fundaciones, sindicatos, patronal, ayuntamientos, etc.), tanto para jóvenes como para adultos.

Es evidente sin embargo que muchos más adultos podrían beneficiarse de una orientación académica y para numerosos jóvenes sería necesario que se diese una orientación profesional mejor que la que actualmente se ofrece.

La Orientación Profesional en la juventud debe implicar una comprensión de los intereses, personalidad, expectativas de los jóvenes, etc., así como una cierta experiencia en el campo del trabajo y del rol que éste juega en la vida de las personas, y todo esto más que una toma de decisiones prematura en el ámbito de una carrera académica y/o profesional.

En los sistemas más efectivos de orientación escolar y profesional deben éstas fusionarse en el momento en el que el joven o el adulto decide las materias a estudiar, o los cursos a seguir para obtener las cualificaciones que le conduzcan a la profesión a escoger.

Así como la información y el consejo orientador propuesto pueden revestir modalidades distintas, son muchos los jóvenes y también los adultos que poseen fuentes de información de modo informal, en el momento de tomar decisiones en el ámbito de la carrera profesional (amigos, familiares, vecinos, compañeros, etc.) y otras personas que a veces juegan un rol más importante que el centro orientador).

En colectivos estables y en períodos de pleno empleo se pueden usar estos procedimientos que se revelan perfectamente ajustados, pero en el contexto actual de desempleo estructural y de cambios rápidos ello se puede traducir a veces en una limitación injusta de las oportunidades personales, lo que afecta especialmente a los jóvenes desfavorecidos o marginados que carecen a veces de fuentes orientadoras bien informadas y objetivas.

Una orientación de calidad tiene potencialmente un rol muy importante que jugar en función del acceso a una formación eficaz o al mercado de trabajo.

Una orientación con efectividad puede hacer progresar la igualdad de oportunidades en función de la etnia, sexo, contexto socioeconómico, etc., si ella está bien delimitada. Existe sin embargo un riesgo continuo que es el de refuerzo de estereotipos negativos, y el hecho de la intervención de orientadores mal preparados o poco reflexivos.

Si se quiere acceder a sujetos marginados es preciso recurrir a otros métodos orientadores como el consejo grupal, o a ciertas actividades de información intensiva mediante la familia.

Uno de los centros de interés de la Orientación en su acepción global es que a sus «clientes» sea cual fuese su edad, debe ayudárseles a ser responsables de su futuro, de hacer 
elecciones y de decidir sobre la base de lo que ellos saben de sí mismos y de los refuerzos que le son dados.

La mejor orientación tiene por objeto no sólo lograr que la persona se inserte bien en un empleo o en un programa formativo que le conviene, sino también el de facilitar la adquisición de competencias para el futuro.

Generalmente es en el frente a frente con su «cliente» y con el análisis de sus necesidades como se ejerce la responsabilidad del orientador, pero esa situación no es tan factible como parece.

Los clientes tienen el derecho de exigir a sus consejeros una orientación independiente e imparcial pero ante esto puede haber conflicto de intereses por ejemplo cuando el Centro de enseñanza que dispone de orientador compite con otros establecimientos, o cuando los poderes públicos cuidan que una política de empleo muy delimitada sea considerada como prioritaria, delante de los clientes, en un número elevado.

\section{Servicios de Orientación Profesional}

En la mayoría de los países desarrollados existe desde hace tiempo una división de funciones entre los Ministerios de Educación y/o de Trabajo y Empleo en el ámbito de la Orientación Profesional. Si la planificación y coordinación son buenas entre ambos organismos puede funcionar la interacción entre éstos.

A veces la existencia de otros Ministerios vinculados con este ámbito como pueden ser el de Asuntos Culturales, de Investigación, Ciencia, Asuntos Sociales, etc., generan dificultades añadidas para llevar a efecto una política congruente de orientación.

En otros casos además de los Ministerios mencionados la enseñanza y el trabajo es administrado también por Estados, Comunidades Autónomas, Administraciones Locales (Ayuntamientos y Diputaciones) y otras autoridades locales (Agencias y Oficinas de Empleo).

La gran diversidad de modalidades de políticas de formación y de empleo tiene una incidencia destacada sobre los modos de organizar la Orientación Profesional que se comienza practicando en el sistema educativo y que se continúa con el empleo y en la formación de un modo continuo.

Los agentes encargados de planificar y administrar las políticas de educación y de trabajo no tienen siembre los mismos objetivos y provienen originariamente de culturas distintas. Los especialistas en educación se interesan en general por las necesidades y la evolución de la persona, mientras que los encargados del empleo y de las cuestiones de trabajo se preocupan básicamente por las necesidades de los empresarios, por el mercado laboral y por la economía en su conjunto.

Se considera a veces que estas preocupaciones diferentes generan antinomias de tal modo que los especialistas en educación son reacios a admitir que se debe preparar a los jóvenes para jugar un rol económico y los gestores de empleo que se interesan especialmente por la colocación, expresan su descontento y frustración frente a la incapacidad aparente del sistema educativo de aportar a las personas los tipos de cualificaciones requeridas y que se estima que la economía tiene necesidad de éstas. Las frustraciones se producen sobre todo en países que no disponen de una tradición profunda de partenariado social, como existe 
por ejemplo en Alemania donde la empresa tiene una gran tradición de trabajo productivo hecho en común.

En los sistemas menos coherentes las situaciones diferentes hechas en la orientación escolar y profesional parecen estar institucionalizadas por la separación entre los Ministerios de Educación y Trabajo, y donde la Formación Profesional queda de una forma débil entre ambas instituciones.

La posición ambigua de la Orientación Profesional representa que en la mayoría de los países de la OCDE ésta está asegurada por tres tipos distintos de organismos, donde cada uno tiene unos puntos fuertes y sus propias debilidades. Son los siguientes:

\section{a) Centros educativos}

La gran ventaja del uso de las escuelas y colegios como establecimientos que facilitan orientación es que los profesores y orientadores conocen y comprenden a los jóvenes que ellos asesoran y que las posibilidades de enseñanza les son familiares. Los inconvenientes radican en que los docentes a veces desconocen las potencialidades y actitudes de los jóvenes, las necesidades del mercado laboral y otros aspectos del mundo externo a las instituciones educativas, y que no están en situación de ofrecer consejos sociolaborales a la juventud que finalizó su escolaridad.

\section{b) Organizaciones del mercado laboral}

Los puntos fuertes de estas entidades que pueden estar administradas por los poderes públicos, centros formativos o de colocación, o dirigidas por los agentes sociales (patronal o sindicatos) que siguen el modelo de partenariado social, son que la orientación es accesible a los jóvenes que finalizaron sus estudios y que los orientadores ponen en estrecho contacto con el mercado laboral. Su principal punto débil es que éstos poseen un conocimiento restringido de sus elecciones educativas, que hacen un largo camino para su colocación y que primen los intereses de las empresas. Corren el riesgo también de no conceder mucho realce a las necesidades e intereses de los empresarios y no dar mucho realce a las necesidades y capacidades de la persona, lo que representa especialmente en el caso de los jóvenes que la formación y la colocación no sean adaptadas a ellos.

\section{c) Agencias públicas o privadas de empleo}

Tienen la posibilidad de que pueden garantizar la conexión entre los Centros docentes y los empresarios y también orientar a los jóvenes que ya no están en el sistema educativo. Estos ni están hipotecados por los establecimientos educativos que pueden desenvolver al máximo los puestos escolares disponibles ni por los empresarios que buscan sencillamente en ellos, en muchas ocasiones, una mano de obra más barata, y por ello los destinatarios tienen una buena oportunidad de obtener una actuación orientadora imparcial.

La principal debilidad del método de contratación laboral de los jóvenes es que puede desembocar en el doble empleo y en la ineficacia y provisionalidad ocupacional. Por otra banda si las agencias cobran a los clientes o a las familias, donde los recursos son insuficientes corren el riesgo de no poder beneficiarse de las ayudas para la contratación laboral de los jóvenes.

A pesar del sistema de prestaciones y ayudas para la inserción laboral de la juventud es posible constatar que muchos de los sistemas nacionales de orientación se caracterizan por 
la incoherencia, las lagunas y los cabalgares inútiles que expresan la inexistencia de un sistema válido y fiable.

La solución debe venir dada por una planificación de las conexiones más eficaces, sin embargo las dificultades son elevadas en ciertos países por la existencia de diferencias de mentalidad entre los Centros de enseñanza y los que ejercen sus actividades en el sistema laboral.

Las diversas medidas a emprender en el ámbito de la coordinación entre el sistema educativo y el laboral precisan de tiempo para madurar y son costosas a corto plazo. Además en varios países europeos la financiación de la Orientación está restringida actualmente lo mismo que otras inversiones públicas.

Es preciso afirmar no obstante que una Orientación Profesional de calidad mediante Servicios adecuados es beneficiosa para los propios jóvenes, para el empresario, para los Centros de enseñanza y para los poderes públicos.

Para que los Servicios Orientadores sean de elevada calidad es preciso vincular los valores, los métodos y las prácticas formativas y laborales.

\section{Acciones orientadoras desde la perspectiva profesional}

Watts y colaboradores (1994) en el informe sobre Orientación Escolar y Profesional en la Unión Europea describen varias tareas distintas que pueden realizarse en las escuelas, en las agencias y servicios para el empleo. Entre ellas podemos considerar las siguientes:

\section{$\mathbf{1}^{\mathrm{a}}$. Información Académica}

Representa aportar datos sobre materias curriculares, programas formativos, planes de estudio, etc. Se trata también de informar a los Centros docentes de cuáles son los tipos de programas donde las empresas y las personas tienen necesidad pero aún no están disponibles.

\section{$2^{a}$. Evaluación}

Consiste esencialmente en la formulación de diagnósticos sobre la adecuación entre las potencialidades de una persona y diferentes alternativas con empleo de instrumentos formales o informales: tests, exámenes, entrevistas, etc.

\section{$3^{\mathrm{a}}$. Orientación}

Ayuda a las personas a concienciarse de lo que piensan sobre diversas situaciones educativas y laborales, de las elecciones que tienen que hacer, académicas y profesionales, y de las consecuencias posibles de las distintas elecciones y tomas de decisión a realizar.

\section{$4^{\mathrm{a}}$. Consejo}

Se basan en propuestas fundamentadas sobre el conocimiento y experiencia del orientador respecto a diversas realidades y situaciones escolares y/o laborales.

\section{5 . Información profesional}

Se trata de aportar datos al mundo de trabajo mediante debates, talleres, juegos de rol, de simulaciones de entrevista, de visita de expertos o de representantes de las empresas de la zona a los Centros escolares, de tareas de iniciación a la práctica y al 
trabajo activo, de visitas a centros laborales para observar «in situ» la realidad ocupacional, etc.

Muchas de estas actividades sirven para ayudar a la persona en la elaboración del curriculum vitae, para la redacción de cartas de presentación profesional por iniciativa propia o demandada por las empresas, para el entrenamiento en la observación, en la entrevista, etc., recursos de gran utilidad para la inserción laboral de la juventud, ya que ayudan a desenvolver conocimientos y competencias para hacer buenas elecciones profesionales que garanticen el éxito en las transiciones académicas y/o ocupacionales.

\section{$6^{\text {a }}$. Colocación}

Consiste en ayudar a los jóvenes a obtener un empleo, a acceder a cursos de formación ocupacional, organizar seminarios de observación del trabajo o de iniciación práctica en diferentes entornos laborales, etc.

\section{$7^{\text {a }}$. Defensa de los intereses de los destinatarios}

Estriba en tratar directamente con los centros de empleo para informarlos de ciertas personas que tienen el riesgo de ser excluidas social y laboralmente o tropiezan con obstáculos en su búsqueda de una formación o empleo.

\section{$8^{\text {a }}$. Seguimiento}

Pretende entrar en contacto con los antiguos clientes para conocer su evolución profesional, observar sus necesidades actuales y saber si precisan ayuda complementaria.

La tipología de actividades enumerada varía de unos países a otros y así la información laboral es una exigencia básica de tal manera que los servicios y las agencias de empleo suelen estar bien equipados en este sector en muchos de ellos, mientras que la Orientación Profesional realizada en los Centros docentes suele mostrar serias lagunas en este ámbito.

La enumeración de tareas expuestas no es exhaustiva y así hay escuelas que ofertan además otras actividades en sus programas formativos (elección de materias optativas, aprendizaje de técnicas de estudio, empleo de recursos de orientación personal y social, etc.).

Por otro lado los Orientadores y los profesores tutores tienen como función relevante ayudar a los alumnos a pasar sin choques bruscos de un nivel de enseñanza a otro, mediante programas de preparación, y garantizar la relación con otros tramos educativos.

\section{La Orientación integrada en el currículo escolar}

Un sistema orientador efectivo es sin duda el de carácter formativo que estimula a los alumnos a comprender y a informarse de la realidad que los rodea, en el sentido por ejemplo de conseguir una información sobre las profesiones, la educación preventiva para la salud, sexo y drogas, las técnicas de estudio y trabajo intelectual, el conocimiento y autoexploración de sí mismo, las competencias de resolución de problemas y toma de decisiones y las cuestiones referentes a la información y orientación profesional.

En Inglaterra el Consejo Nacional de Programas recomendó en 1991 que los Centros educativos debían proponer a los alumnos acceder a una orientación personalizadora, enfa- 
tizando en la educación secundaria la experiencia directa del mundo laboral, el acceso a fuentes informativas actualizadas referentes a las ramas existentes en el ámbito de la enseñanza general o profesional en el campo formativo y laboral, la posibilidad de elaborar un dossier personal expresando los logros obtenidos y la construcción de un plan de acción personal.

Entre los elementos educativos a desarrollar, figuran los métodos de estudio, el plan de carrera académico-profesional, la identificación de competencias, intereses y valores, el dominio de técnicas de exploración sobre el empleo, un plan de visitas a las instituciones laborales, una semana sobre «información de profesiones » y un programa de actividades prácticas de iniciación al trabajo.

\section{La elección académico-profesional}

Tradicionalmente en la mayoría de los tránsitos de un nivel a otro de la enseñanza, o de la escuela a la vida profesional activa, se generaban de una manera más o menos automática, con un acceder masivo de un sistema a otro, de tal manera que en esa situación en las que las acciones no se hacían bien, la orientación académica y/o profesional no era imprescindible.

En el contexto actual, debido a que los sistemas educativos y profesionales son más complejos, cobra importancia la orientación educativa y profesional y los estudiantes tratan de diseñar itinerarios personales y profesionales propios en el que incluyen actividades de formación, trabajo, de posible desempleo, etc.

El paso de la escuela a la vida laboral hace que la orientación profesional tenga un rol esencial a desempeñar.

Es aconsejable, que los alumnos realicen programas de estudios generales y equilibrados considerando las elecciones académicas y profesionales ulteriores y a la vez los intereses y aspiraciones específicas. En este sentido se debe orientar a la juventud a encontrar puertas abiertas en el futuro debiéndose hacer elecciones reversibles en una época donde aún no cuentan siempre con la madurez necesaria para elegir.

El sistema educativo debe asesorar al alumnado a estudiar materias que correspondan con sus intereses, preferencias y capacidades.

La dicotomía entre formación general y específica es inevitable en una situación donde los jóvenes deben prepararse para un futuro que es difícil de prever. La Orientación juega aquí un rol relevante para superar el conflicto entre una base cultural común y la necesaria diversidad, facilitando la elección, especialmente en el momento en que la juventud sale de la escolaridad obligatoria donde el programa es sustantivamente igual para todos y en el futuro deben escoger una opción especializada en función de sus itinerarios personales.

En determinados sistemas educativos existen distintos tipos de instituciones docentes en función de la modalidad de estudios, de carácter más bien general o profesional.

La Orientación Educativa descansa en gran medida en la función de elección diferenciada entre las decisiones propias de los escolares y las que son tomadas por otros para ellos.

En los sistemas educativos más selectivos habitualmente las decisiones son tomadas por los alumnos, sobre todo al final de la escolaridad obligatoria. 
En países de la Unión Europea como Alemania, Austria o Italia se recomiendan distintos tipos de educación secundaria a los alumnos y a sus familias, partiendo de aptitudes y intereses de ellos y del conjunto de los resultados escolares, pero en todo caso la Orientación puede tener una función esencial.

En Finlandia, por ejemplo, la juventud dispone de un ámbito amplio de elección de modalidades de enseñanza y de formación y el alumnado es estimulado a escoger con total autonomía, de acuerdo con sus intereses y expectativas.

De todas maneras, la concurrencia para entrar en ciertos Centros educativos es viva y eso conlleva que ciertos estudiantes deben reformular sus proyectos. La Orientación tiene un gran rol a desempeñar ayudando a los jóvenes a adoptar decisiones realistas.

Por otro lado en determinados países de la Unión Europea existen teóricamente posibilidades de elección académica por los escolares, pero está limitada a veces, incluso en ocasiones disimuladamente, por las capacidades de acogida de las instituciones más solicitadas.

Watts y colaboradores (1994) observaron que las decisiones cruciales de carácter académico son tomadas por el alumno cuando se trata de una repartición «vertical» entre los diferentes niveles de enseñanza, mientras que las elecciones horizontales entre distintas ramas tienen mucha más oportunidades de ser hechas por el alumnado, con o sin orientación.

Sin embargo la elección en horizontal puede tener también una repercusión jerárquica en la que los estudiantes no son siempre conscientes.

\section{El tránsito de un nivel educativo a otro}

Los pasos importantes no representan solamente una elección, si no que expresan el crecimiento y la evolución así como la superación obligada de una etapa. De esta manera los escolares que pasan de la educación primaria al primer ciclo de enseñanza secundaria cambian nítidamente de estatus.

Si el alumnado correspondiente es apoyado a lo largo de este cambio por un programa orientador bien concebido la transición tiene muchas posibilidades de ser un éxito.

El escolar ve una nueva situación confirmada y accede a lo largo de ella con una progresión y una evolución educativa sobre la cual podrán apoyarse posteriormente para avanzar más.

La transición con éxito representa habitualmente considerar dos elementos, la preparación de ella y su puesta en marcha, pero los sistemas escolares no siempre lo tienen en cuenta.

\section{Preparación}

En varios países, entre ellos España, el tránsito entre la educación primaria y la secundaria obligatoria es a los 12 años y ello conlleva preparar este cambio. En Finlandia el paso del primer al segundo nivel de escuela polivalente es a los 13 años. 
En cualquiera caso se observa en esta etapa una evolución importante que precisa una preparación específica y un programa común destinado a capacitar a los alumnos para la fase siguiente a través, por ejemplo, de las actividades siguientes:

$1^{\mathrm{a}}$. Las escuelas de enseñanza secundaria organizan una jornada de puertas abiertas para los escolares y sus padres o les autorizan en determinados momentos a ver como funciona el Centro educativo correspondiente.

$2^{\mathrm{a}}$. En el último curso escolar el alumnado de enseñanza primaria puede visitar a los escolares de educación secundaria obligatoria con sus profesores tutores para elegir el Centro docente o para conocer la institución anticipadamente en la que van ingresar.

$3^{\mathrm{a}}$. Los escolares de primer curso de enseñanza secundaria obligatoria organizan una presentación especial de acogida de los nuevos compañeros de su antigua escuela primaria que muchos ya conocen, después de la primera visitas de ellos.

$4^{\mathrm{a}}$. Los Orientadores y profesores tutores del establecimiento de enseñanza secundaria pueden visitar a sus futuros educandos en la escuela de enseñanza primaria, acompañados de los alumnos del primer curso procedentes de ella y que superaron con éxito la transición, con objeto de describirles el nuevo Colegio, responder a sus preguntas y tranquilizar las eventuales inquietudes.

$5^{\mathrm{a}}$. Folletos y prospectos referentes a los Centros de enseñanza secundaria pueden distribuirse entre los escolares del último curso de educación primaria, y también a sus padres, generando intercambios entre maestros y alumnos, o entrevistas con los padres.

El tránsito de los Centros docentes de enseñanza secundaria a la Universidad o a otra modalidad de enseñanza representa en la mayoría de los países de la Unión Europea otra gran perspectiva de transformación y cambios, y la orientación ofrecida a los jóvenes para prepararse para éstos varía considerablemente entre ellos.

En Inglaterra las universidades en general organizan unas jornadas de puertas abiertas para los futuros estudiantes, y en algunos Centros universitarios los alumnos de primer curso son estimulados a comunicarse con los nuevos antes de comenzar el curso para darles consejos sobre lo que deben hacer en determinadas situaciones y ofrecerles una amistad en las primeras semanas.

En Austria y Italia las Universidades tienen contactos con los Centros de enseñanza secundaria, los profesores universitarios se trasladan a éstos para explicar el funcionamiento en su establecimiento de los diversos programas a los estudiantes potenciales y para animarlos a elegir.

Estas visitas responden a un objetivo doble en el proyecto de orientación: posibilitar el tránsito de aquellos estudiantes que ya decidieron los estudios que pretenden seguir y ayudar a los dudosos a adoptar una decisión.

\section{Implementación}

En muchos países de la Unión Europea las tasas de abandono de los estudios universitarios son elevadas. En múltiples casos los estudiantes se sirven de la Universidad para no 
inscribirse en los registros de desempleo, mientras buscan un trabajo o adoptan otras decisiones con vistas a su futuro, de tal manera que no materializan la elección de la carrera elegida. En estos casos se puede deducir que la orientación académica y profesional de los estudiantes no alcanza su objetivo, o aquéllos que tenían necesidad de asesoramiento no lo recibieron.

Esta realidad en la toma de decisiones por el alumnado cuesta muy caro al país y enseña que la orientación ofertada deja bastante que desear tanto cuantitativa como cualitativamente.

Los métodos de Orientación que consisten en ayudar a los alumnos a integrarse en los nuevos estudios varían en los diferentes países de la Unión Europea y también entre las instituciones educativas. Existen documentos y folletos destinados a informar a los estudiantes y a su familia y en muchos Centros educativos comienzan sus actividades con una jornada de acogida, orientada a familiarizar a los nuevos alumnos con la institución correspondiente y a localizar sus instalaciones y servicios (aulas, biblioteca, etc.).

Se promueve también en el nuevo alumnado el contacto con otros estudiantes para posibilitar su integración social y académica.

En el ámbito de los Centros educativos los padres de los alumnos nuevos son invitados a veces a visitarlos y a participar en talleres y reuniones.

Estas actividades están concebidas para proveerlos de experiencias e información suficientes sobre la institución escolar, y facilitar a las familias de los discentes consejos para ayudarlos en el curso de sus estudios, para posibilitar la convivencia entre los padres de los escolares, etc.

Como estrategias y técnicas de orientación se utilizan la entrevista personal, el asesoramiento grupal, los debates, role-playing, el brainstorming, etc.

\section{La transición del ámbito académico a la vida profesional}

El paso de la vida escolar al mundo activo conlleva la modificación de actividades de la juventud y suponen el tránsito de la escuela al empleo o a la búsqueda del mismo. La etapa de transición es un período de tiempo en el cual una promoción de jóvenes pasa de la asistencia a los Centros educativos a tiempo completo a una actividad en el mercado laboral, o a la búsqueda de la inserción profesional.

La amplitud de este período varía en función de los sistemas educativos y formativos y de los empleos disponibles. En la mayor parte de los países esta etapa abarca de los 1630 años.

La situación actual del mercado de empleo para los jóvenes exige el máximo de esfuerzos por parte de los Servicios de Orientación Profesional.

En todos los países de la Unión Europea la tasa de empleo de los menores de 25 años es superior a la tasa de desempleo general. Un joven europeo de promedio sobre cinco está sin trabajo y ello representa una profunda inquietud en cada uno de los países de Europa. 
Tasas de desempleo de los jóvenes menores de 25 años en los países de la Unión Europea (Eurostat, 1999).

\begin{tabular}{|l|c|c|}
\hline \multirow{2}{*}{ Países } & \multicolumn{2}{|c|}{ Tasa de desempleo } \\
\cline { 2 - 3 } & $\begin{array}{c}\text { Tasas de desempleo de } \\
\text { los menores de 25 años } \\
\text { (en porcentajes) }\end{array}$ & $\begin{array}{c}\text { Tasas de desempleo, } \\
\text { en general } \\
\text { (Datos en porcentajes) }\end{array}$ \\
\hline Alemania & 10,1 & 9,3 \\
\hline Austria & 6,3 & 4,4 \\
\hline Bélgica & 21,6 & 8,6 \\
\hline Dinamarca & 6,3 & 4,2 \\
\hline España & 33,9 & 18,2 \\
\hline Finlandia & 23,6 & 11,0 \\
\hline Francia & 26,8 & 11,8 \\
\hline Gran Bretaña & 13,5 & 6,2 \\
\hline Grecia & 19,1 & 9,8 \\
\hline Irlanda & 12,0 & 8,6 \\
\hline Italia & 32,5 & 12,3 \\
\hline Luxemburgo & 5,8 & 2,2 \\
\hline Países Bajos & 6,6 & 3,7 \\
\hline Portugal & 8,9 & 4,5 \\
\hline Suecia & 14,8 & 7,5 \\
\hline
\end{tabular}

La transición de la educación a la vida profesional representa tener en cuenta diversas modalidades de formación y acceso al empleo, donde la elección es un factor de singular importancia.

En los países industrializados el período de transición se alarga y tiene una naturaleza más compleja, abarcando una simultaneidad o alternancia entre etapas de formación de ejercicio profesional y de desempleo.

En varios países de la Unión Europea existen actividades organizadas de iniciación práctica al trabajo, a veces mediante el desarrollo del sistema de partenariado entre los Centros docentes y las empresas, especialmente a través del sistema de prácticas en instituciones laborales.

Los jóvenes realizan habitualmente una serie de tareas en las empresas sin ser considerados como salarios. En varias ocasiones esta preparación es la pasarela que conduce a un empleo estable y en otras es una aproximación al conocimiento real de una profesión.

Entre las modalidades de iniciación a la vida laboral se pueden mencionar las siguientes: 
$1^{\circ}$. Observación del trabajo, donde los alumnos en prácticas ven las actividades que efectúan los trabajadores en la empresa y su rol en la misma.

$2^{\circ}$. Visitas de empresa. Los alumnos observan como está organizado un centro laboral, y cómo se trabaja, métodos y productos de la institución profesional respectiva, etc.

$3^{\circ}$. La simulación que abarca el role-playing y los juegos de roles y de acciones diversas hechos en la escuela pero basándose en cuadros realistas.

$4^{\circ}$. La monitoría donde a través de un sistema tutorial los alumnos observan el trabajo en la empresa, discuten los problemas laborales, etc., con un tutor responsable de su supervisión profesional.

$5^{\circ}$. Creación de mini-empresas por el alumnado en la propia escuela en conexión con el entorno laboral, desenvolviendo acciones de autoempleo.

$6^{\circ}$. Formación práctica en las empresas para los profesores, a través de períodos temporales de permanencia laboral en las mismas. Esto repercute en la propia formación y en la enseñanza que imparte a los estudiantes.

$7^{\circ}$. Realizar prácticas de formación profesional en otros países efectuadas mediante programas europeos como Leonardo. Consisten en iniciativas innovadoras de elevado interés en la tareas de transición a la vida profesional activa por el lado de los jóvenes.

\section{Conclusiones}

En el contexto de la Unión Europea a Orientación Profesional de la juventud debe pretender alcanzar una serie de necesidades que hacen referencia en primer lugar a una información académica detallada de los sistemas de formación y especialmente de la profesional.

Asimismo se deben dar a los jóvenes ayuda y consejos referentes a la situación actual y futura previsible del mercado de trabajo y ofertas de la formación profesional en diferentes áreas territoriales y países.

La Orientación Profesional debe apoyar las competencias demandadas sobre las ofertas de empleo, especialmente las sociales, las prácticas profesionales, el trabajo en equipo, el espíritu de iniciativa, el dominio de lenguas extranjeras, sobre todo el inglés, las habilidades informáticas y la actitud de movilidad socio-laboral.

La realidad presente del mercado de trabajo para la juventud en los países que integran la Unión Europea exige el máximo de esfuerzos por parte de los servicios de orientación profesional y especialmente por los Orientadores de los mismos como agentes de cambio y dinamización socio-laboral.

\section{Referencias bibliográficas}

Alverca, C. y outros (1994). Determinação das necessidades em Orientação Escolar y Profissional de diferentes grupos alvo de jovens entre 15 y 27 años en Portugal. O caso da península de Setubal. Berlin: CEDEFOP. 
Arthur, M. B. y otros (1999). The new careers: individual action and economic change. Londres: Sage.

Bundesanstalt für Arbeit (1999). Europaische kouferenz der Nationalen Ressourcen Centren für Berufsberatung. Nuremberg.

CEDEFOP (1998). Conseil et Orientation Professionnelle tout au long de la vie. Tesalónica.

CERI (1996). Mapping the future. Young people and Career Guidance. París: OCDE.

Gysbers, N. y Henderson, P. (1997). Comprehensive guidance programs that work. Il. Greenboro, N.C.:E.R.I.C./C.A.S.S.

Gysbers, N. C. y Henderson, P. (1998). Developing and Managing your school guidance program. Alexandria, VA: AACD.

Jato, Y. (1998). La Formación Profesional en el contexto europeo. Nuevos desafios y tendencias. Barcelona: Estel.

OCDE (1998). Analyse des politiques de l'éducation. París.

OCDE (1999). Evaluation des performances et des politiques. París.

Raban, A. (1997). The role of employers in Guidance for current and future students. A European perspective. Cambridge: University Careers Service.

Scottish Office Education Departament (1995). Views on Scottish Education and international comparisons. Edimburg.

Sobrado, L. (1999). Orientación e Intervención Sociolaboral. Barcelona: Estel.

Sobrado, L. (2000a). Glosario de Orientación Escolar y Profesional. Santiago de Compostela: AIOSP/Universidade de Santiago de Compostela.

Sobrado, L. (2000b). Orientación Profesional: Diagnóstico e Intervención Sociolaboral. Barcelona: Estel.

Super, D. y Sverko, B. (1995). Life, roles, values and careers. San Francisco: Jossey-Bass Pub.

Watts, A. G. (1996). Rethinking careers and vocational guidance. London: Routledge.

Watts, A. G. (2201). «L'education en Orientación pour les jeunes». En J. Guichard y otros. «Eduquer en Orientation: enjeux et perspectives». Actes du colloque international. INETOP, Paris.

Watts, A. G. y colaboradores (1994). Educational and Vocational Guidance in the European Community. Luxemburg: Office for Publications of the European Communities.

Watts, A. G. y Van Esbroeck, R. (1999). Nouvelles competences pour un avenir different. Bruselas: FEDORA/UUB University Press.

Fecha de recepción: 9-I-02

Fecha de revisión: 31-I-02

Fecha de aceptación: 5-II-02 